\title{
UNIVERSITY OF LEEDS
}

This is a repository copy of Electron temperature and mechanisms of hot carrier generation in quantum cascade lasers .

White Rose Research Online URL for this paper:

http://eprints.whiterose.ac.uk/1689/

\section{Article:}

Harrison, P., Indjin, D. and Kelsall, R.W. (2002) Electron temperature and mechanisms of hot carrier generation in quantum cascade lasers. Journal of Applied Physics, 92 (11). pp. 6921-6923. ISSN 1089-7550

https://doi.org/10.1063/1.1517747

\section{Reuse}

See Attached

\section{Takedown}

If you consider content in White Rose Research Online to be in breach of UK law, please notify us by emailing eprints@whiterose.ac.uk including the URL of the record and the reason for the withdrawal request. 


\title{
Electron temperature and mechanisms of hot carrier generation in quantum cascade lasers
}

\author{
P. Harrison, ${ }^{\text {a) }}$ D. Indjin, and R. W. Kelsall \\ School of Electronic and Electrical Engineering, University of Leeds, Leeds LS2 9JT, United Kingdom
}

(Received 22 May 2002; accepted 4 September 2002)

\begin{abstract}
A technique for calculating the temperature of the nonequilibrium electron distribution functions in general quantum well intersubband devices is presented. Two recent $\mathrm{GaAs} / \mathrm{Ga}_{1-x} \mathrm{Al}_{x} \mathrm{As}$ quantum cascade laser designs are considered as illustrative examples of the kinetic energy balance method. It is shown that at low current densities the electron temperature recovers the expected physical limit of the lattice temperature, and that it is also a function of current density and the quantised energy level structure of the device. The results of the calculations show that the electron temperature $T_{e}$ can be approximated as a linear function of the lattice temperature $T_{l}$ and current density $J$, of the form $T_{e}=T_{l}+\alpha_{e-l} J$, where $\alpha_{e-l}$ is a coupling constant $\left(\sim 6-7 \mathrm{~K} / \mathrm{kA} \mathrm{cm}^{-2}\right.$ for the devices studied here) which is fixed for a particular device. (C) 2002 American Institute of Physics.

[DOI: $10.1063 / 1.1517747$ ]
\end{abstract}

The rapid development of the quantum cascade laser as an application of the mid- and far-infrared radiative intersubband transitions available in semiconductor heterostructures has been impressive and continues to be prominent in the applied physics literature. However, in addition, quantum cascade lasers have also given new insight into more physical aspects of the quantum mechanics of electron transport, see for example, Ref. 1. In particular, an earlier work by Harrison ${ }^{2}$ deduced that: (i) the electron distributions in quantum cascade lasers are thermalized, i.e., the energy distribution of electrons in each subband can be described by a Fermi-Dirac distribution function and (ii) the electron temperature for each subband is the same. Both of these conclusions were substantiated by experimental measurements of the electroluminescence spectra of superlattice cascade lasers ${ }^{3}$ and Monte Carlo simulations. ${ }^{4}$ While Monte Carlo simulations can be valuable for investigating the physics of intersubband devices on a microscopic scale, they are difficult to implement, and are very demanding computationally. In this work an alternative more accessible technique for calculating the average electron temperature throughout the device is developed, which can be applied to all intersubband electron- or hole-based quantum devices.

The approach adopted is based on energy balance. Given that any intersubband device will always reach an equilibrium under continuous operating conditions, then the rate at which the electron (or hole) distributions gain kinetic energy (relative to the particular subband minimum) through scattering will balance with the rate at which they lose kinetic energy to the lattice. In the steady state, the lattice temperature will also reach an equilibrium. The lattice temperature itself will always be controlled by the device design, packaging, and cooling systems-in developmental laboratories many measurements are made under cryogenic conditions which give the lattice temperature more specifically.

\footnotetext{
a) Author to whom correspondence should be addressed; electronic mail: p.harrison@physics.org
}

It is acknowledged that, in areas of high current density and intense phonon production, the phonon distribution may not be in equilibrium with the lattice, however, these effects are likely to be small. The aim of this work is to understand and demonstrate a technique to calculate global effects-the electron temperature in the device as a single number.

Consider the two instances of intersubband scattering as in Figs. 1(a) and 1(b). In Fig. 1(a) the subband separation $E_{i}-E_{f}$ between the initial electron state $|i\rangle$ and the final state $|f\rangle$ is greater than the dominant phonon energy [in III-V's the polar longitudinal optical (LO) phonon], hence any phonon emission event produces a carrier in the lower subband with an increased kinetic energy. This energy adds to the total kinetic energy of the electron distributions, and through the subpicosecond intrasubband carrier-carrier scattering events, ${ }^{2}$ is quickly absorbed by the distributions as they rethermalize. Bi-intrasubband carrier-carrier scattering (of the form $|j\rangle|g\rangle \rightarrow|j\rangle|g\rangle)$ serves to further redistribute this energy throughout the set of subbands within the quantum device and to equalize the temperatures of the distributions. ${ }^{2}$ In Fig. 1(b) the subband separation $E_{i}-E_{f}$ is less than the phonon energy, hence a scattering event from the upper to the lower subband reduces the total kinetic energy of the electrons.

In both cases, the change in the total kinetic energy of the electron distributions can be written as: $\Delta E=E_{i}-E_{f}$ $-E_{\mathrm{LO}}$, where $\Delta E>0$ should be interpreted as an increase and $\Delta E<0$ a decrease in this total energy. If there are $n_{i}$ carriers in the initial state and the LO phonon transitions have associated scattering rates of $1 / \tau_{i f}^{\mathrm{em}}$ and $1 / \tau_{i f}^{\text {abs. }}$ for emission and absorption processes, respectively, then the net kinetic energy generation rate from intersubband scattering is

$$
\sum_{f \neq i} \sum_{i}\left[\frac{n_{i}}{\tau_{i f}^{\mathrm{em} .}}\left(E_{i}-E_{f}-E_{\mathrm{LO}}\right)+\frac{n_{i}}{\tau_{i f}^{\mathrm{abs} .}}\left(E_{i}-E_{f}+E_{\mathrm{LO}}\right)\right]
$$

where the indices on the summations imply over all initial and final states in the quantum system. 


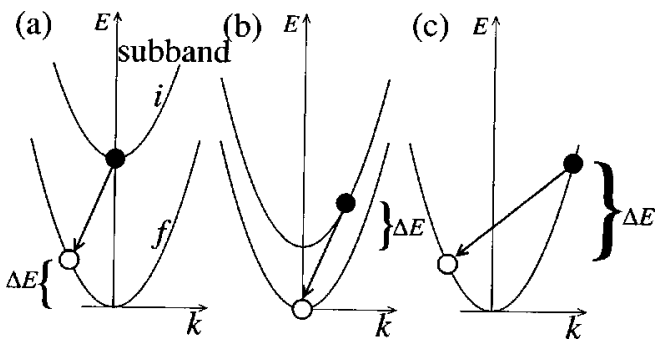

FIG. 1. Schematic diagram illustrating inelastic (polar optic phonon) intersubband scattering processes for subband separations (a) greater than and (b) less than the LO phonon energy. (c) illustrates an inelastic intrasubband scattering process.

One of the main features of quantum cascade lasers is the bridging (injector) regions between the active layers. These regions serve to remove the electrons from one active region and supply them, at the correct energy, to the next active region. There is, however, another important role that they provide - the opportunity for hot (high kinetic energy) carriers to cool. The injector-collector regions can be optimized to do this by designing the separation of the subbands to be less than the LO phonon energy. Hence, they encourage the carriers to scatter as in Fig. 1(b), thus losing kinetic energy and cooling the distribution. Another mechanism that contributes to this cooling is illustrated in Fig. 1(c)intrasubband phonon emission. Such transitions lead to a decrease in the energy by an amount $E_{\mathrm{LO}}$, again if this has a scattering rate of $1 / \tau_{i i}^{\mathrm{em}}$, then the corresponding kinetic energy loss rate from intrasubband scattering is

$$
\sum_{i}\left[\frac{n_{i}}{\tau_{i i}^{\mathrm{em}}} E_{\mathrm{LO}}+\frac{n_{i}}{\tau_{i i}^{\mathrm{abs}}}\left(-E_{\mathrm{LO}}\right)\right],
$$

where $1 / \tau_{i i}^{\text {abs. }}$ accounts for intrasubband phonon reabsorption, which reduces the above energy loss rate by $-E_{\mathrm{LO}}$.

Carrier-carrier (electron-electron or hole-hole) scattering events are described as elastic, which means that the total energy of the particles before the event is the same as that after. However, intersubband electron-electron transitions do convert potential energy into kinetic energy (or vice versa), which from the viewpoint of this work would lead to an increase (decrease) in the total kinetic energy of a subband population. Note, the potential energy as defined here includes the quantized component of the kinetic energy.

It has been argued that at equilibrium the intersubband generation rate is equal to the intrasubband loss rate. This can be written succinctly as

$$
\Delta=\sum_{\text {em.,abs., } c-c} \sum_{f} \sum_{i} \frac{n_{i}}{\tau_{i f}}\left(E_{i}-E_{f}+\delta E\right)=0,
$$

where the change in energy $\delta E$ is equal to $-E_{\mathrm{LO}}$ for phonon emission (em.), $+E_{\mathrm{LO}}$ for absorption (abs.), and zero for carrier-carrier $(c-c)$ scattering.

Equation (1) is a kinetic energy balance equation. Now the scattering rates in Eq. (1) are functions of the subband populations $n_{i}$ and the electron temperatures [if the electron temperature is too low, the number of intrasubband scattering events, as in Fig. 1(c) is too small and the kinetic energy equation cannot be balanced].

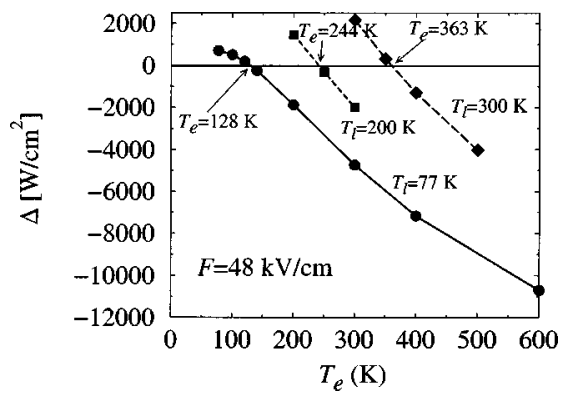

FIG. 2. The calculated value of the kinetic energy generation rate $\Delta$ as a function of the electron temperature and for several different lattice temperatures for the $x=0.33$ device at the operating field of $48 \mathrm{kV} \mathrm{cm}^{-1}$.

The scattering rates $1 / \tau$ and subband carrier densities $n_{i}$ in multilevel quantum cascade structures, can be calculated using the self-consistent scattering theory approach of Donovan et al..$^{5}$ and later developed (and validated by comparison with current-voltage curves and laser output characteristics, such as threshold current, gain and temperature dependence) by Indjin et al. ${ }^{6}$ The procedure now is to vary the electron temperature (which, as argued above, is assumed to be the same for all subbands) until the kinetic energy balance equation is satisfied self consistently.

For the purpose of this illustration two significant GaAs/ $\mathrm{AlGaAs}$ quantum cascade laser designs were employed. One of the lasers was the first reported $\mathrm{GaAs} / \mathrm{Ga}_{1-x} \mathrm{Al}_{x} \mathrm{As}$ laser ${ }^{7}$ (with $x=0.33$ ) which has been extensively studied ${ }^{1,2,4}$ and adapted still further, see for example Refs. 8 and 9. The second design is more recent ${ }^{10}$ and was the first GaAs $/ \mathrm{Ga}_{1-x} \mathrm{Al}_{x}$ As $(x=0.45)$ device to lase at room (lattice) temperature, again this device has been the attention of further studies. ${ }^{11}$ For specific details of the layer structures and doping profiles, see the original reports. ${ }^{7,10}$

Figure 2 illustrates the method for the calculation of the electron temperature. Following our standard procedure for deducing the carrier densities and lifetimes across all levels of the device (the model includes all states across an injector/ active region/collector, i.e., 1 1/2 periods, which involves 15 levels in total), the self-consistent iteration is extended to include the effect of the electron temperature. The data in Fig. 2 show the variation of the net kinetic energy generation rate $\Delta$ with the electron temperature $T_{e}$ for the $x=0.33$ cascade laser. The solutions for the electron temperatures occur when $\Delta=0$. Detailed study of the contributions to the electron temperature highlight two interesting points: first that phonon absorption mechanisms are important even at low $(77 \mathrm{~K})$ lattice temperatures, and second that, for lattice temperatures above $50 \mathrm{~K}$, electron-electron scattering is not an important electron heating mechanism (accounting for less than $1 \%$ of the total heat generation rate) in these midinfrared lasers.

Figure 3 summarizes the electron temperature $T_{e}$ (given by the circles) as a function of the current density $J$ through both the quantum cascade lasers. It can be seen that at very low current densities (low input powers) the model recovers the expected physical limit of the electron temperature being equal to the lattice temperature, but as the current density is increased, the electron temperature increases too-in agree- 


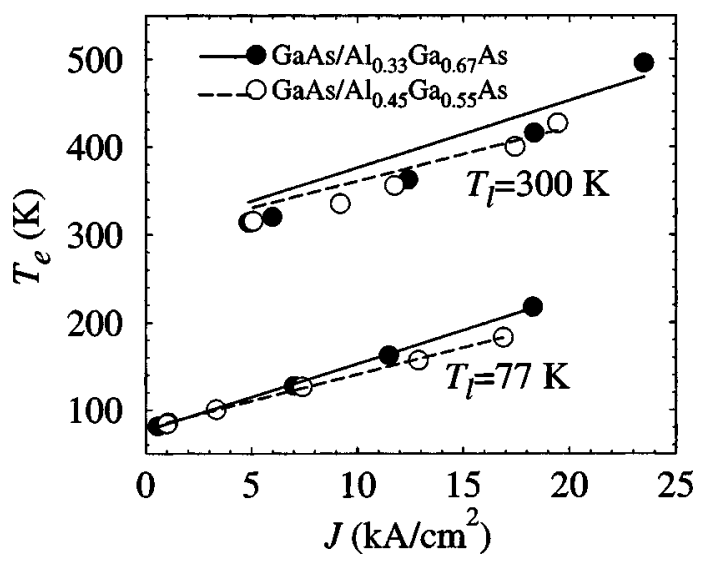

FIG. 3. The calculated electron temperature as a function of current density for the two different quantum cascade laser structures considered and at lattice temperatures of 77 and $300 \mathrm{~K}$.

ment with experiment. ${ }^{3}$ For both devices at a lattice temperature of $77 \mathrm{~K}$, the relationship between $T_{e}$ and $J$ turned out to be linear

$$
T_{e}=T_{l}+\alpha_{e-l} J,
$$

where the electron-lattice $(e-l)$ coupling constant $\alpha_{e-l}$ was deduced from the straight line fits to the data (as on Fig. 3) and had values of 7.6 and $6.1 \mathrm{~K} /\left(\mathrm{kA} \mathrm{cm}^{-2}\right)$ for the $x$ $=0.33$ and $x=0.45$ devices, respectively. At the higher lattice temperature of $300 \mathrm{~K}$, the calculations showed that there is some deviation from this linear relationship, however, Eq. (2) (with the same coupling constants) is still a reasonable approximation, as can be seen by the straight lines on Fig. 3 .

It is expected that the technique developed in this work will now allow the important aspect of the energy within the electron distributions of intersubband devices to be considered in the design and optimization stages of development. Such knowledge will be crucial in solving the problems of temperature dependence of the lasing characteristics of the new generations of very long wavelength (Terahertz) quantum cascade lasers reported recently. ${ }^{12,13}$ Application of the techniques presented here to the Terahertz laser of Köhler et al. ${ }^{12}$ has shown that the coupling constant $\alpha_{e-l}$ is much larger $\left(\sim 47 \mathrm{~K} / \mathrm{kA} \mathrm{cm}^{-2}\right)$ than in the mid-infrared devices above. This is a consequence of the increased role of electron-electron scattering in this device operating at low $(5-50 \mathrm{~K})$ lattice temperatures and demonstrates the greater sensitivity of the electron distribution functions to the injection current - $\mathrm{a}$ fact that will have to be controlled, either through reduced threshold currents or improved injector design, if high temperature operation of Terahertz lasers is to be achieved.

In summary, a general technique has been presented which allows the temperature of the nonequilibrium electron distributions in quantum intersubband devices to be calculated as a function of all the device operating parameters, such as the electric field, the current density, and the lattice temperature. In the illustrative examples of quantum cascade lasers it was found that the electron temperature has a near linear dependence on the input current density, although the coupling constant (slope) is different for different device designs.

The authors would like to thank EPSRC (U.K.) for financial support and Z. Ikonić for useful discussions.

${ }^{1}$ R. C. Iotti and F. Rossi, Phys. Rev. Lett. 87, 146603 (2001).

${ }^{2}$ P. Harrison, Appl. Phys. Lett. 75, 2800 (1999).

${ }^{3}$ M. Troccoli, G. Scamarcio, V. Spagnolo, A. Trediccuci, C. Gmachl, F. Capasso, D. L. Sivco, and M. Striccoli, Appl. Phys. Lett. 77, 1088 (2000).

${ }^{4}$ R. C. Iotti and F. Rossi, Appl. Phys. Lett. 78, 2902 (2001).

${ }^{5}$ K. Donovan, P. Harrison, and R. W. Kelsall, J. Appl. Phys. 89, 3084 (2001).

${ }^{6}$ D. Indjin, P. Harrison, R. W. Kelsall, and Z. Ikonic, J. Appl. Phys. 91, 9019 (2002).

${ }^{7}$ C. Sirtori, P. Kruck, S. Barbieri, P. Collot, J. Nagle, M. Beck, J. Faist, and U. Oesterle, Appl. Phys. Lett. 73, 3486 (1998).

${ }^{8}$ L. R. Wilson, J. W. Cockburn, M. J. Steer, D. A. Cavder, and M. S. Skolnick, Appl. Phys. Lett. 78, 413 (2001).

${ }^{9}$ S. Barbieri, C. Sirtori, H. Page, M. Stellmacher, and J. Nagle, Appl. Phys. Lett. 78, 282 (2001).

${ }^{10}$ H. Page, C. Backer, A. Roberston, G. Glastre, V. Ortiz, and C. Sirtori, Appl. Phys. Lett. 78, 3529 (2001).

${ }^{11}$ D. Indjin, P. Harrison, R. W. Kelsall, and Z. Ikonic, Appl. Phys. Lett. 81, 400 (2002).

${ }^{12}$ R. Köhler, A. Tredicucci, F. Beltram, H. E. Beere, E. H. Linfield, A. G. Davies, D. A. Ritchie, R. C. Iotti, and F. Rossi, Nature (London) 417, 156 (2002).

${ }^{13}$ M. Rochat, L. Ajili, H. Willenberg, J. Faist, H. Beere, G. Davies, E. Linfield, and D. Ritchie, Appl. Phys. Lett. 81, 1381 (2002). 